\title{
FORMACIÓN CIUDADANA Y AUTORREGULACIÓN: CONCEPTOS CLAVES PARA LA PRÁCTICA EDUCATIVA
}

\author{
CIVILIAN EDUCATION AND \\ SELF-REGULATION: KEY CONCEPTS FOR AN \\ EDUCATIONAL PRACTICE
}

Marianne Beuchat Reichardt

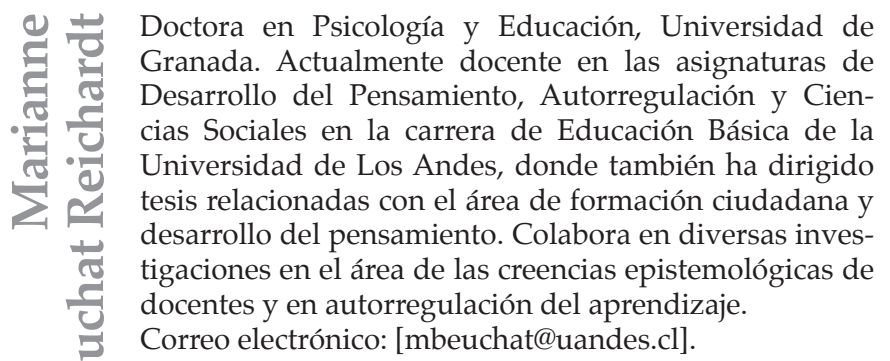

\section{RESUMEN}

Se expone la relación de los conceptos de autorregulación del aprendizaje y formación ciudadana en el marco imperante de formar ciudadanos socialmente activos.

La autorregulación se entiende como una forma de configurar, entre otros, pensamiento y afectos (Trías y Huerta, 2009). Es en la capacidad 
del «hacer», donde se produce la relación con la educación. Este hacer es posible gracias a la dimensión social presente en la vida del ser humano; aquí se enraíza la formación ciudadana. No se nace siendo ciudadano, sino que se aprende a ser ciudadano.

Palabras clave: autorregulación del aprendizaje; educación cívica; formación ciudadana.

\section{ABSTRACT}

This presentation examines the relationship between self-regulation in learning and civilian education within the framework of forming citizens who will actively participate in society.

Self-regulation is understood as a way to configure - among other elements - thoughts and affects (Trías y Huerta, 2009). It is in the capacity for «doing» where the connection to education is established since it is the social dimension of human life that requires «doing» and wherein civilian education is rooted. One is not born a citizen but learns how to be a citizen.

Keywords: self-regulated learning; political education; civilian education.

\section{INTRODUCCIÓN}

Podemos situar los conceptos de formación ciudadana y autorregulación como retos de la educación en el mundo global y en la sociedad del conocimiento. Entonces, ¿en qué medida articular la formación ciudadana con la autorregulación?

La formación ciudadana se percibe como una preocupación constante y de carácter urgente por la necesidad imperante que posee toda nación de formar ciudadanos participativos y que contribuyan al bien común. La formación ciudadana involucra a la educación que responde de por sí — debido a su origen y su función- a la dimensión social del 
hombre. Aristóteles (1979) señala que el «hombre es un ser social» por naturaleza, que necesita de otros para vivir. La persona, por lo tanto, vive con otras personas; precisa de la sociedad, requiere de la vida en comunidad (entendida como una agrupación de personas que mantienen un lazo de tipo espiritual y que comparten una cultura y modo de vida en común). De acuerdo con Cordua (2012), quien sigue esta línea de pensamiento, la educación considera el sentido de la formación de una personalidad compleja y matizada, de una preparación para lograr una coexistencia social civilizada para el desempeño de una ciudadanía consciente y responsable.

Como conducta, la autorregulación se refiere directamente al comportamiento que sustenta un determinado individuo. A medida que la persona adquiere habilidades de autorregulación, se presentan cambios en su conducta, que lo beneficiarán en todos los aspectos: se vuelve una persona más responsable, con mayor auto-dominio y desarrolla diferentes aptitudes como, por ejemplo, la propuesta de metas para los distintos objetivos que se le presentan o desea alcanzar. Cabe la pregunta, ¿se aprende a ser autorregulado?

\section{AUTORREGULACIÓN Y APRENDIZAJE}

Para llegar a un aprendizaje significativo y profundo - no sólo en el ámbito académico, sino en todos los aspectos vitales-, es necesario que el aprendiz sea un individuo autorregulado, que se sepa protagonista de su aprendizaje y practique habilidades que lo beneficien para alcanzar sus objetivos. Si consigue el auto-dominio, la persona logrará fijar metas reales en tiempos determinados, y practicará todo lo que requiere el alcance de una meta. Trías señala que:

[...] la autorregulación del aprendizaje no debe entenderse como una característica, rasgo o cualidad personal. Por el contrario, puede considerarse como una forma de configurar pensamientos, afectos, conductas en interacción con los elementos que el contexto proporciona y con la finalidad de cumplir los objetivos personales (Trías y Huertas, 2009, p. 1). 
Por otra parte, Zimmerman (1989); Zimmerman y MartínezPons (1986), y Pintrich (1995), señalan la autorregulación como un proceso personal para alcanzar habilidades que orienten su propia conducta:

La autorregulación puede definirse como la capacidad que una persona adquiere para orientar su propia conducta. En el contexto del aprendizaje, la autorregulación consiste básicamente en formularse metas concretas, planificar actividades para el logro de esas metas, monitorear el desempeño durante la ejecución de tales actividades, evaluarse a sí mismo de forma continua, de acuerdo con las metas y criterios fijados, $y$, por último, valorar el producto del proceso de aprendizaje (López-Vargas, Hederich-Martínez y Camargo-Uribe, 2011, p. 7).

Para alcanzar la autorregulación es fundamental el conocimiento propio: estar al tanto de las propias cualidades, fortalezas, debilidades y los defectos. Si se advierten dichos aspectos personales, alcanzar un objetivo será un proceso más realista y se ideará un plan personal a partir de las propias características.

La autorregulación como competencia incluye entonces que el individuo se conozca, conozca su propio proceso de aprendizaje, programe conscientemente sus estrategias de aprendizaje, de memoria, de solución de problemas y toma de decisiones, y se exprese con autonomía al aprender. Otro de los aspectos es la capacidad de transferir esos procesos y habilidades a otras situaciones o contextos (Pineda, 2011, p. 4).

Es en la capacidad de transferencia donde se inserta la autorregulación en el ámbito de la actuación humana. Si la educación fuera un mero concepto estático, carecería de sentido su objetivo. Cuando se habla de educar o bien del concepto de educación, no se hace referencia, en estricto rigor, a lo que el hombre es, sino a lo que hace.

La actuación humana se constituye en dos tipos de finalidades objetivas: obrar y hacer. Obrar se refiere a la acción que culmina en sí misma. Hacer, por el contrario, implica un proceso, es decir, una sucesión de movimientos que desembocan en un resultado final (Altarejos y Naval, 2004). 
El saber práctico implica lo moral, lo cual es capaz de orientar el obrar (Volpi, 2008) y también la voluntad. La deliberación racional de lo que se ha de hacer no puede entenderse únicamente como deducción racional, porque la razón que determina la voluntad, no es la razón teórica, sino la práctica. La razón teórica alcanza el bien sólo como verdadero y no como bueno (Arregui y Choza, 1995). Se llega por medio de la razón práctica a alcanzar el bien que reconoce. $\mathrm{Al}$ conocer el bien como fin, se produce una tendencia hacia éste. El dinamismo que esto genera es lo que convierte a la razón teórica en práctica (Arregui y Choza, 1995).

Chemello (2000) plantea la necesidad de que el hombre aprenda a vivir bien. Aristóteles (1988, p. 144) afirma: «El hombre feliz vive bien y obra bien». Entonces, ¿cómo vivir bien? Altarejos y Naval (2004) responden que la educación es la encargada de formar hábitos operativos buenos, que caracterizan el vivir y obrar bien. Estos hábitos operativos contemplan a su vez la dimensión social del hombre, la cual dentro de sus aspectos considera la formación o educación ciudadana.

Altarejos y Naval (2004) sostienen que la educación sólo es posible gracias a la dimensión social presente en la vida del hombre y que, para desarrollar esta dimensión - que se encuentra en potencia-, es necesaria la educación. Una persona no educada, no puede vivir en sociedad. Esta dimensión social, aspecto integrante de toda educación, implica por una parte la formación ciudadana y por otra, la autorregulación.

De acuerdo a lo anterior, tanto docente como estudiante son mediadores activos que, al emplear el pensamiento crítico, combinan la práctica académica y la reflexión con la finalidad de educar ciudadanos reflexivos y activos (Carmona, 2008).

Una persona nace desconociendo - desde luego- su propio proceso de aprendizaje como, por ejemplo: programar conscientemente las estrategias de aprendizaje para solucionar los problemas, tomar decisiones en forma autónoma y participar activamente en la sociedad con la finalidad de contribuir al bien común de la misma. 
Estos procesos deben monitorearse constantemente; son procesos proactivos. La enseñanza de las habilidades operativas para que la persona desarrolle sus potencialidades en diferentes dimensiones, se inicia con un aprendizaje observacional; más tarde viene la imitación; se continúa con la internalización de las habilidades y, por último, la autorregulación que las lleva a la práctica.

Ahora bien, la autorregulación centrada en el aprendizaje promueve específicamente cambios en las conductas que beneficiarán al estudiante en todos los aspectos: se vuelve una persona más responsable, con mayor autodominio, va desarrollando diferentes aptitudes y se fijará metas para los distintos objetivos que se le presenten. Los estudiantes autorregulados poseen estrategias cognitivas, metacognitivas, motivacionales y de apoyo, y requieren llevarlas a la práctica para dirigir su aprendizaje, construyendo así sus conocimientos de forma significativa. De esta manera, regulan y controlan intencionalmente todo el proceso, conocen sus habilidades, los conocimientos que poseen, saben qué deben hacer para formarse, han aprendido a identificar sus conductas de estudio, ajustan sus conductas y actividades a las demandas de estudio, están motivados para aprender y son capaces de regular su motivación.

En palabras simples: la autorregulación es un proceso interno que incorpora factores exteriores, que permiten a la persona adquirir habilidades que la beneficien para el logro de objetivos. Existen factores totalmente relacionados con éste y, sin ellos, no se alcanza una autorregulación como tal. Algunos son: cognición, metacognición, motivación, intervención, entre otros, sin descartar que planificar y fijar objetivos previamente es fundamental para conseguir el objetivo que se desea alcanzar, teniendo en cuenta las características personales que pueden intervenir en dicho proceso.

Al hacer referencia al aprendizaje o enseñanza, hay que tener en cuenta que cada persona posee características diferentes y capacidades distintas, que la hacen ser original frente a los demás individuos. Si se observa en un aula de clases de cualquier nivel, frente a una enseñanza específica - ya sea de un contenido o de otro-, existen diversas maneras de registrar dicha información; esto demuestra que cada alumno posee su estilo de aprendizaje, 
en donde practica sus habilidades concretas para adquirir nuevos conocimientos.

Las estrategias de aprendizaje, aparecen a nivel cognitivo, actitudinal y procedimental; son las capacidades del individuo para planificar cómo alcanzar su objetivo: se proponen metas y se idea un plan de acción para obtener el logro. No hay que descartar - como ya se ha señalado- que el auto-conocimiento es fundamental en este proceso ya que, a partir de él, la persona aplica estrategias que estén a su alcance y sean reales. La metacognición trata el autoconocimiento de la actividad cognitiva de la persona, abordando el control de ésta, de manera que se planifique lo que se llevará a cabo para el alcance de sus objetivos, se supervisa durante la ejecución y se evalúan los resultados obtenidos en relación con la meta propuesta. A fin de cuentas, la metacognición es una cognición de la cognición. Cabe destacar que al tomar conciencia de los contenidos y de las estrategias utilizadas para su obtención, éstas son resultado de una reflexión realizada durante el proceso de aprendizaje. Para un buen manejo metacognitivo por parte de quien aprende, es necesario desarrollar el nivel del saber sobre el hacer.

En este proceso de aprendizaje, hay que tener en cuenta que los estudiantes no están solos: siempre se acompañarán de sus profesores que son los encargados de guiar dicho aprendizaje, y lograr que el estudiante obtenga resultados positivos y que, día a día, vaya progresando. Muchas veces, al hablar de autorregulación, se piensa en un proceso personal e individual; sin embargo, para desarrollar habilidades de autorregulación es necesaria la ayuda de otro que monitoree y guíe en todo este camino. De acuerdo con ello, el docente cumple un rol primordial al abordar dichas tareas.

Otro aspecto importante para el desarrollo de competencias en este proceso es el transcurso de la autorregulación y el auto-control de la conducta. Su relevancia se debe a que el individuo autorregulado ha de ser capaz de dominar su comportamiento y hacerse cargo de su aprendizaje; el estudiante se responsabiliza de lo que aprende y su capacidad de auto-control de la conducta debe favorecer lo que va construyendo. Reconocer la situación problemática, conocer las variables que pueden causar las conductas incontrolables, formular 
un plan para prevenir el mal comportamiento, la realización del plan establecido y luego la evaluación de éste (López, 1992), son acciones que desarrollan estrategias de autorregulación del aprendizaje, para así incrementar los conocimientos de los estudiantes sobre éstas. De esta manera se promueven competencias de estudio mediante procesos de formación que permitan enfrentar desafíos académicos de la manera más competente posible.

Chemello (2000) alude a que el agente principal de la educación es el propio educando, quien se perfecciona realizando actos ordenados a alcanzar virtudes intelectuales o morales. Sin estos actos propios, la ayuda del educador no tendría sentido, no conduciría a nada. En este contexto, este orden de virtudes intelectuales o morales puede propiciar y dar sentido a la formación ciudadana, concepto que se procede a describir a continuación.

\section{FORMACIÓN CIUDADANA}

El concepto de ciudadanía, ha sido formulado a partir de la Grecia clásica. Esto constituye un hecho diferenciador de otras civilizaciones de la Antigüedad, como la babilonia, egipcia, asiria y caldea, entre otras: estas civilizaciones jamás se habrían imaginado que el hombre pudiese gobernarse a sí mismo y pudiese dictar y elaborar leyes de comportamiento. La ciudadanía es, entonces, un modo de inserción en la sociedad.

Por consiguiente, puede definirse la ciudadanía como la capacidad política para intervenir en la «cosa pública». De acuerdo a lo señalado en las diferentes legislaciones, son ciudadanos quienes han cumplido una determinada edad, junto a otros requisitos que varían de estado a estado. En casi todos los países, la ciudadanía se manifiesta por medio del derecho de sufragio en un régimen político democrático que implica una forma de gobierno y una estructura socioeconómica, de valores, actitudes y conductas democráticas.

Cabe decir que la democracia es el sistema político más aceptado en el mundo. Su importancia radica en que reconoce la dignidad de 
las personas. Este sistema de gobierno es considerado la mejor forma de organización sociopolítica que garantiza el respeto, el ejercicio y la promoción de los derechos humanos. Se caracteriza por ser constitucionalista, por establecer el bien común como fin del estado, por ser el gobierno de la mayoría respetando a la minoría y porque propicia el pluralismo ideológico, incentiva la libertad política y posibilita una competencia pacífica hacia el poder.

El poder del Estado se distribuye en diferentes órganos, se reconoce la autonomía de los cuerpos intermedios y, finalmente, se permite la vigencia de un estado de derecho. Los principales principios de la democracia son: respetar, promover y garantizar los derechos humanos, además de la autodeterminación del pueblo o la soberanía popular (Squella, 2000).

En suma, se concluye que el concepto de ciudadanía posee como elementos básicos: los derechos, la pertenencia y la participación activa en la Res publica (Aristóteles, 1932). La ciudadanía se concibe como:

[...] la inserción y la expresión participativa y perteneciente al individuo humano en los espacios públicos con derechos, deberes y accesos comunitarios, civiles, políticos, sociales, económicos y culturales a objeto de hacer realidad la democracia participativa (Boyoga, Hernández y Santana, 2006, p. 11).

El hombre por tanto, de la mano con la idea de perfección y la de ser un individuo social por naturaleza, se perfecciona con la ayuda de otros, y por sí solo contribuye al bien común de la sociedad de la que es parte; esto sólo se alcanza mediante el ejercicio de la participación ciudadana, es decir, a través de una formación ciudadana. Aquí radica, por tanto, la importancia de la educación en la formación ciudadana, ya que es, a través suyo, que se logra y se hace efectiva la participación ciudadana en miras al bien común de la sociedad de la que es parte todo individuo.

En suma, educar no sólo implica la enseñanza de contenidos, sino que conduce al educando a constituirse - mediante la perfección de sus potencias- en una mejor persona. La educación envuelve 
la dimensión social del hombre y con ella la formación ciudadana, actividad integradora de la persona con su entorno social. Además, esta acción educativa contempla formar a un individuo reflexivo, capaz de desenvolverse de manera autónoma y proactiva en la comunidad del que es parte integrante.

De acuerdo a Nussbaum (2005) las habilidades que se requieren para el cultivo de la humanidad son: por una parte un examen crítico, tanto de uno mismo como de las propias tradiciones; la capacidad de verse a sí mismo, no como ciudadano perteneciente a una región o un grupo sino, también, y sobre todo, como ser vinculado a los demás seres humanos por lazos de reconocimiento y de mutua preocupación; otra de las capacidades es la imaginación narrativa que pretende desarrollar la empatía o la capacidad de pensar cómo sería estar en el lugar de otra persona, ser un lector inteligente de su historia y comprender las emociones, deseos y anhelos que alguien así pudiera experimentar (Vilafranca y Buxarrais, 2009).

Desde una perspectiva filosófica-antropológica, la formación ciudadana implica una exigencia ética en la que los individuos, al estar en estrecha relación, trabajan conjuntamente en construir y conservar dichas relaciones. Esto es simplemente lo propio de su esencia constitutiva, es decir: es lo propio de su naturaleza social el desarrollo, por medio del perfeccionamiento, de todas sus potencias y del ejercicio de una vida virtuosa.

Cano (2005) plantea que no basta sólo con educar al niño para sí, ni para su país, ni para vivir: es necesario educarlo para y con los demás, para el mundo y para convivir en sociedad. La educación comienza en la casa, y luego en el colegio, lo que aprende en estas dos partes se refleja en su vida diaria, pero esta formación no se logra sin actividades especiales. Para ello resulta necesario crear un buen ambiente de clase en el colegio, desarrollando hábitos que contribuyan al desarrollo de un pensamiento reflexivo, a la propia autorregulación y a la formación ciudadana; estos aspectos se asientan en principios, se enmarcan en la base de los derechos humanos, se relacionan con la vida cotidiana y, además, implican un desarrollo de habilidades y conocimientos para convivir con los demás en democracia. 
El objetivo es comprometerse con los demás, participar con respeto y confianza, con la intención de aceptar a los otros y comprenderlos, para así poder formar un equipo, compartiendo de manera respetuosa. Vivir conviviendo con los demás, es ir transformándose en ciudadano. No se nace siendo ciudadano, sino que se aprende en la casa, en el colegio, en la calle, en el trabajo, con los amigos, por lo que la tarea no es solamente de los profesores.

Procurar ser un mejor ciudadano requiere desarrollar un conjunto de habilidades, conocimientos, disposiciones y actitudes favorables al desarrollo de la ciudadanía. En distintos países, la orientación prevista a nivel curricular consiste en explicitar en los programas no sólo el conocimiento relacionado con la formación ciudadana, sino enfatizar el desarrollo de habilidades, como la capacidad de formular opiniones fundamentadas, propiciar actitudes, entre otras, de solidaridad y respeto. Por esto, la formación ciudadana es parte integral de la educación de los alumnos, extendiéndose a todos los niveles. Para lograrlo se intentan diferenciar dos tipos de contenidos y habilidades en el currículo: uno relacionado con el desenvolvimiento de los alumnos, ligado a los bienes materiales y simbólicos (es decir, a ser un consumidor bien informado con capacidad crítica), y por otro lado, lo tocante a la integración política y la convivencia democrática, es decir, a una participación política. Éstos forman parte de los objetivos fundamentales transversales que incitan la valoración de la democracia y el pluralismo, el cuidado del medio ambiente, la igualdad de ambos géneros, el respeto a los derechos humanos y a la diversidad cultural. Al ser objetivos transversales se trabajan en distintas asignaturas, haciendo de la formación ciudadana no sólo un concepto a aprender, sino un conjunto de habilidades que deben ser aprendidas durante los años escolares (Cerda et al., 1994).

En síntesis, educar es más que instruir, es humanizar, y por lo tanto, la definición de los fines de la educación depende, en gran medida, de la concepción que se sustente sobre el ser humano (Gómez, 2001; Gordillo, 2006; Touriño, 2009).

Una educación integral tendrá en cuenta las distintas dimensiones humanas y los diferentes ámbitos en los que es posible y deseable educar a las personas. Conocer, manejar, valorar y participar es útil 
para identificar algunas finalidades sustantivas de la acción educativa. Educar para conocer supone recuperar la relevancia del conocimiento como finalidad de la actividad educativa. Educar para realizar implica orientarse medularmente hacia el aprendizaje de la interacción. Educar para valorar significa reconocer la importancia de lo axiológico en la formación del individuo y su desarrollo del juicio moral. Educar para participar conlleva el propiciar escenarios en los que sea posible aprender a tomar decisiones que involucren la vida social. Estas cuatro finalidades son relevantes para orientar acciones educativas encaminadas a posibilitar una educación para la ciudadanía (Gordillo, 2006).

La formación para la convivencia ciudadana aparece, entonces, como una responsabilidad compartida y derivada de la sociedad como factor de desarrollo educativo y de la misma educación como factor de desarrollo social, que debe cumplir las exigencias del carácter axiológico de la educación, en lo que corresponde a la formación para la convivencia pacífica en un marco legal territorializado de relación con el otro. El desarrollo cívico es el objetivo de la formación para la convivencia ciudadana.

La educación ciudadana tiene como objetivo central formar personas con habilidades básicas para la vida democrática y el desarrollo. Ello implica capacitar a las personas para que sean autónomas y capaces de formular propuestas conscientes de su identidad, sus derechos y responsabilidades, de tal manera que participen activamente en la vida cotidiana, cívica y pública, en la renovación de instituciones y en la toma de decisiones que involucren mejoras a las condiciones de vida. La educación debe contribuir de una manera especial a la gobernabilidad democrática, preparando personas adecuadamente calificadas y formando ciudadanos arraigados a la cultura cívica democrática, capaces de desarrollar juicios críticos y de ponerlos en práctica desde la niñez.

La construcción de la ciudadanía es un proceso que se genera principalmente desde abajo y que atraviesa todas las esferas de la vida de las personas, de la familia y del Estado; tarea a la que todos estamos llamados a participar de una manera u otra. La ciudadanía supone un conjunto de derechos y deberes que se van articulando en este proceso 
y que posibilita que las personas participen en la toma de decisiones sobre los asuntos que les competen. Es una parte del proceso de socialización, es decir, del proceso mediante el cual una persona se convierte en miembro de una sociedad.

La ciudadanía necesita ciudadanos, personas formadas para la conciencia y el ejercicio de sus derechos fundamentales, seres cuyo valor no radica en formar parte de una colectividad ni en su naturaleza gregaria, sino en su propia identidad personal; individuos, conscientes de que ellos -y no los Estados ni los gobiernos- son los soberanos. Sólo sobre la base de ciudadanos fuertes es posible pensar en sociedades con derechos ciudadanos sólidos. La formación de ciudadanos conscientes de sus derechos y responsabilidades públicas no se agota en los alcances políticos del término, los trasciende.

Las responsabilidades democráticas - no estrictamente políticasincluyen una gran variedad de actividades públicas y sociales. Para el ciudadano común, para aquel que no tiene toda su vida centrada en el tema político, lo cívico y lo civil tienen más elementos en común de lo que habitualmente piensa.

Muchos esfuerzos de educación ciudadana se han frustrado por prestar una atención desproporcionada a los fines frente a los medios. Lo que hace buenos ciudadanos no es el hecho de coincidir en los objetivos de la convivencia social, sino su común respeto a las reglas que posibilitan alcanzarlos y mantenerlos establemente. El tema recurrente de la institucionalidad democrática asoma aquí nuevamente para enfatizar la importancia crítica de su presencia también para el logro de la educación ciudadana. Sólo si los ciudadanos respetan las reglas pueden exigir que éstas sean igualmente respetadas por sus gobernantes. Las reglas no deben concebirse como regalos de los gobernantes, sino como exigencia de los gobernados. El constante ejercicio de esta exigencia es, a fin de cuentas, la mejor expresión de una cultura ciudadana.

Cada persona puede ser un líder. Hay que desprender el liderazgo de su connotación excepcional y entenderlo también como parte de una materia educativa, esto es, susceptible de descubrirse y desarrollarse. Una sociedad democrática requiere de líderes. 
La formación ciudadana desarrolla el sentido de pertenencia, convivencia y el interés por la participación social (Maiztegui, 2007). Su principal objetivo es fomentar los valores y conocimientos necesarios para desarrollarse en la vida pública y colaborar en un posible cambio social; ayuda a creer en el espíritu crítico y transformador, que propicia la práctica de los derechos y los deberes ciudadanos. De acuerdo a Nussbaum (2005), el ciudadano del mundo es una persona con un gran desarrollo de pensamiento crítico; es éticamente sensible y empático.

La precariedad en la formación ciudadana de los niños y jóvenes como personas capaces de desempeñarse idóneamente en la vida democrática, en los diversos contextos - personales, familiares, comunitarios, ciudadanos, culturales y productivos-, invita a repensar la propia práctica educativa y pedagógica.

De acuerdo a un enfoque de educación liberal, el docente y el alumno dejan de ser pasivos y se convierten en mediadores activos para encontrar su propia teoría y el nuevo conocimiento empleando el pensamiento crítico. La educación debiera abocarse a la tarea de activar, en cada estudiante, una mente independiente y producir una comunidad que verdaderamente razone en conjunto sobre un problema y no simplemente intercambie argumentos (Vilafranca y Buxarrais, 2009).

Ortega y Mínguez (2001) sostienen la trascendencia de la experiencia del valor. Isaacs (1991) explica cómo adquiere importancia el educador en la medida en que actúe de manera virtuosa, pues las virtudes tienen un fin en la felicidad y no en sí mismas. Chemello (2000) afirma que es necesaria la inclinación afectiva del sujeto a las virtudes. Esto implica que es el propio educando quien desea y se propone realizar estos fines en su vida. ¿Cómo llegar a esto? Descubriendo el valor que implica, en la dimensión social del ser humano, el significado de vivir con otro. Por medio de la razón se descubren los argumentos que dan sentido a los comportamientos virtuosos, a las acciones de héroes, desde el ciudadano anónimo al destacado político. El aporte que otorga la historia, la filosofía, y en 
general las ciencias sociales y las humanidades, a la reflexión de lo que significa vivir en comunidad, facilita en el educando un mayor nivel de adhesión de las competencias requeridas para compartir una cultura cívica.

Uno de los escenarios donde se asimila esta cultura cívica es la institución educativa. Es allí donde se aprende a construir con el otro y confiar en él, para convivir civilizadamente. De ahí la urgencia en la formación de competencias, no sólo en la gestión pedagógica, sino también en la administrativa de las instituciones educativas para atender — cada vez con mayor eficiencia- las demandas educativas y formativas de la población para una mayor comprensión social en el ejercicio de los derechos democráticos y de ciudadanía por parte de la población escolar. El constante ejercicio de este respeto es la mejor expresión de una cultura ciudadana. Entonces, ¿existe una relación estrecha entre autorregulación y formación ciudadana?

\section{AUTORREGULACIÓN Y FORMACIÓN CIUDADANA}

Una persona que reflexiona sobre su hacer y pensar, y que se fija objetivos y metas, probablemente tomará distancia y analizará su rol en la sociedad.

Cuando se piensa de manera analítica, cuando se controla de forma intencional el propio aprendizaje, practicando habilidades para alcanzar el objetivo propuesto, se interioriza la dimensión social de la persona, dimensión relacionada con la vida cotidiana y expresada, por ejemplo, en la formulación de opiniones fundamentadas y en actitudes arraigadas en una cultura cívica-democrática.

Lo manifestado, entonces, no será sólo un acuerdo verbal, sino que éste se encauzará hacia resultados, producto de una reflexión.

La pregunta eje de la autorregulación del aprendizaje es cómo resolver mejor la situación a la que el individuo personalmente se enfrenta. 
La respuesta conlleva la realización de preguntas acerca del problema y de cómo solucionarlo.

Por lo general, cuando los profesores plantean un problema o una situación, han sido ellos mismos, previamente, quienes han formulado la pregunta. Una parte esencial del proceso de autorregulación es que el propio estudiante tome conciencia que existen preguntas que es fundamental abordar: éste es, en sí, el reconocimiento de problemas. Ordinariamente, las personas no se preguntan cómo mejorar la convivencia: continúan relacionándose como lo han hecho por siempre.

Al ser consciente de los procesos involucrados en los comportamientos -en relación con los objetivos que surgen o que se han propuesto-, la autorregulación conduce a tomar conciencia, también, de los aspectos constitutivos de la vida diaria. Una pregunta, por ejemplo, de cómo mejorar nuestro entorno surgiría de la situación en la que se ve enfrentada la persona para resolverlo. En otras palabras, la persona autorregulada identifica los problemas y, para ello, requiere de un espacio de aprendizaje que plantee desafíos.

Por tanto, pareciera ser que la autorregulación está estrechamente ligada al pensamiento analítico y responde a una cuestión o a un problema, reflexiona sobre el uso de elementos de razonamiento y lo controla.

Ahora bien, pensar no es tan lineal como podría suponerse. En lo general, pensar en forma analítica es separar los elementos constitutivos que componen el tema, la situación o el problema. La división puede llevar a la comprensión de estas partes en su todo. En ciertos casos se trata de considerar las diferentes alternativas de solución y, con ello, anticiparse a las consecuencias de una decisión determinada.

Se necesita del marco de la autorregulación, ya que en dicho proceso se aprende a establecer objetivos y diseñar el plan concreto de realización. Los objetivos dirigen la conducta y el esfuerzo de la persona en dirección a su consecución, logrando una mayor concentración para analizar los distractores, por ejemplo, que alejan de la tarea. 
En síntesis, la autorregulación facilita el proceso analítico y posibilita una discusión fundamentada en torno a temas de formación ciudadana. Estos temas deberían desarrollarse en la escuela de manera integrada y en las diferentes asignaturas. El sistema educativo requiere pensarse como escuela de ciudadanía, pues es a través de la práctica donde se aprenden las reglas del orden social y donde se aprende a razonar acerca de ellas. Las habilidades necesarias para desempeñarse activamente en la sociedad se sustentan en el propio proceso de conocimiento y autorregulación. Aquí radica la conjunción entre autorregulación y formación ciudadana.

\section{REFERENCIAS}

Altarejos, F. y Naval, C. (2004). Filosofía de la educación. España: Universidad de Navarra.

Aristóteles (1988). Política. Madrid: Gredos.

Aristóteles (1979). Política. Edición bilingüe y traducción por Marías y Araujo. M., Madrid: Instituto de Estudios Políticos.

Aristóteles (1933). Obras completas. Madrid: Edit. Bruno del Amo. Traducción F. Gallech Palés.

Arregui, J.V. y Choza, J. (1995). Filosofía del hombre, una antropología de la intimidad, cuarta edición. Madrid: Ediciones Rialp S.A.

Biblioteca del Congreso Nacional de Chile (s.f.). El ciudadano y la ley. 11 de junio de 2012. Recuperado de http:/ / www.bcn.cl/ ecivica/index_html

Boyoga, N.; Hernández, E. y Santana, C. (2006). Educación ciudadana y democracia: un acercamiento desde la filosofía pedagógica. Bajo palabra: Revista de filosofía, II, época 1 (2006), pp. 6-16. 
Cano, A.M. (2005). Formación ciudadana, estrategias metodológicas para cultivar las competencias ciudadanas, preescolar/primaria. Bogotá: Paulinas.

Carmona G.M. (2008). Hacia una formación docente reflexiva y crítica: fundamentos filosóficos. Revista de teoría y didáctica de las ciencias sociales, XIII (13), pp. 125-146.

Cerda, A.M.; Egaña, M.L.; Magendzo, A.; Cruz, E.S. y Vargas, R. (1994). El complejo camino de la formación ciudadana, una mirada a las prácticas docentes. Madrid: Alianza.

Cordua, C. (2012). La crisis de las humanidades. Revista de Filosofía, 68, pp. 7-9.

Chemello, A.A. (2000). Educación a la felicidad, educación en las virtudes: la importancia de una comprensión adecuada de la naturaleza y función de la virtud. Romae: Pontificia Universitas Sanctae Crucis.

Gómez, G. (2001). Educación para la ciudadanía. Valparaíso: Universidad Adolfo Ibáñez, Instituto de Humanidades.

Gordillo, M.M. (2006). Conocer, valorar, manejar, participar: los fines de la educación para la ciudadanía. Revista iberoamericana de educación, 42, pp. 69-83.

Isaacs, D. (1991). La educación de las virtudes humanas. Pamplona: EUNSA.

Klimenko, O. (2009). La enseñanza de las estrategias cognitivas y metacognitivas como una vía de apoyo para el aprendizaje autónomo en los niños con déficit de atención sostenida. Revista Virtual Universidad Católica del Norte (27), pp. 1-19, 30 de marzo de 2015. Recuperado de http://www.redalyc.org/articulo. oa? id=194215432005 
López-Vargas, O.; Hederich-Martínez, C. y Camargo-Uribe, A. (2011). Estilo cognitivo y logro académico. Revista electrónica Educación y Educadores, 14 (enero-abril), 30 de marzo de 2015. Recuperado de http://www.redalyc.org/articulo. oa?id=83418921005

López, S. (1992). Autorregulación y desarrollo de capacidades que incrementan la coherencia entre juicio y acción. Comunicación, lenguaje y educación, 4 (15), pp. 111-118.

Maiztegui Oñate, C. (2007). La participación como una opción transformadora en los procesos de educación ciudadana. Revista iberoamericana sobre calidad, eficacia y cambio en educación, 5 (4), pp. 144-160, 20 de julio de 2012. Recuperado de http://www. redalyc.org / articulo.oa?id $=55140509$

Nussbaum, M. (2005). El cultivo de la humanidad. Una defensa clásica de la reforma en la educación liberal, trad. J. Pailaya. Barcelona: Paidós.

Ortega, P. y Mínguez, R. (2001). Los valores en la educación. Barcelona: Ariel Educación.

Pineda, I.S. (2011). Calidad del aprendizaje y procesos de metacognición $y$ autorregulación en entornos virtuales $y$ duales en educación superior. Ponencia Facultad de Educación y Ciencias Humanas, Universidad de Córdoba-Colombia, 12 de febrero de 2014. Recuperado de www.virtualeduca.info/ponencias2011/119/ Ponencia

Pintrich, P. (1995). Understanding Self-Regulated Learning. Understanding Self-Regulated Learning, ed. P. Pintrich. San Francisco, CA: Jossey-Bass.

Squella, A. (2002). Introducción al derecho. Santiago: Editorial Jurídica. 
Touriñán, J. (2009). El desarrollo cívico como objetivo: una propuesta pedagógica. Teoría de la educación, (21:1), pp. 129-146.

Trías, D. y Huertas, J.A. (2009). Autorregulación del aprendizaje y comprensión de textos: estudio de intervención. Ciencias psicológicas, 3, pp. 7-16.

Vilafranca, I. y Buxarrais, R.M. (2009). La educación para la ciudadanía en el debate comunitaristas-liberales, Bordón 61 (2), pp. 139-149. España: Universidad de Barcelona. España.

Volpi, F. (2008). Rehabilitación de la filosofía práctica y neoaristotelismo. Navarra: Servicio de Publicaciones de la Universidad de Navarra.

Zimmerman, B.J. (1989). Models of self-regulated learning and academic achievement. Self-Regulated Learning and Academic Achievement: Theory, Research, and Practice, B.J. Zimmerman and D.H. Schunck (eds.). New York: Springer-Verlag.

Zimmerman, B.J. \& Martinez-Pons, M. (1986). Development of a structured interview for assessing students' use of selfregulated learning strategies. American Education Research Journal, 23, pp. 614-628. 